\title{
Archäometrische Analysen von Lengyelkeramik aus Niederösterreich
}

Ângela Carneiro

Kurzfassung:

Dieser Beitrag präsentiert die ersten Ergebnisse zur Untersuchung von Herstellungsprozessen (Rohstoffauswahl, Aufarbeitung und Herkunft sowie Maltechniken) von Keramik der Lengyelkultur aus der Siedlung von Michelstetten, die im Zentralweinviertel des norddanubischen Niederösterreich liegt und nach der Radiokarbon-Methode zwischen 4600 und 4360 a cal. BC datiert wurde. Unter Anwendung verschiedener archäometrischer Verfahren konnte die mineralogische Zusammensetzung von Lengyelkeramik und ihre Malpigmente sowie von Ton- und Lehmmaterialien, die als Rohstoffproben gedient haben, bestimmt werden. Neben einer genauen Charakterisierung der Herstellungssubstanzen prähistorischer Keramik von Michelstetten wurden unterschiedliche Erzeugungsvorgänge, wie Rohstoffauswahl, Art und Qualität der Materialaufarbeitung, Brennverfahren und Maltechniken behandelt, genauso wie Herkunftsfragen diskutiert.

[Archaeometrical analysis of Lengyel pottery from Lower Austria]

Abstract:

This study is about methods used in the production of prehistoric ceramic (origin and selection of raw materials, treatment of fabrics and painting techniques) based on several analysis of ceramic pottery from the site of Michelstetten in the North of Lower Austria dated between 4600-4360 a cal. BC. Using different archaeometrical methods it was possible to know the mineralogical composition of Lengyel pottery, her painting pigments and of the raw material collected near the site. Besides a specific characterisation of the clay materials used in the production of prehistoric pottery from Michelstetten, a number of pottery techniques like selection of raw material, type and quality of fabrics, firing methods and painting techniques will be explained and discussed here as well as questions about the origin of the raw materials.

Keywords: $\quad$ production of neolithic ceramic, Lengyel Culture, Lower Austria

Adress of author: $\quad$ A. Carneiro, Fundação para a Ciência e a Tecnologia, Portugal. E-Mail: Angela.Carneiro07@gmail.com

\section{Einleitung}

Um Auswahl, Art der Aufarbeitung und Herkunft verwendeter Rohstoffe sowie angewendete Brennverfahren und Maltechniken an Lengyelkeramik der Siedlung von Michelstetten (Niederösterreich, 4600-4360 a cal. BC) näher zu kennen, wurden zuerst verschiedene archäologische Untersuchungen an ca. 6.000 Gefäßeinheiten durchgeführt. Danach wurden aus einer gezielten Keramikauswahl 37 Keramikproben aus Michelstetten und 22 Ton- und Lehmmaterialien als Rohstoffproben aus der Umgebung der Siedlung von Michelstetten, unter Anwendung von petrographischen und Schwermineralanalysen zur mineralogischen Bestimmung und zu Herkunftsfragen prähistorischer Keramik und Tonrohstoffen untersucht. Schließlich wurden Röntgendiffraktometrie und Polarisationsmikroskopie zum Erkennen der chemischen $\mathrm{Zu}$ sammensetzung der Malpigmente an 16 weiteren Lengyelkeramikproben aus Michelstetten angewendet.

Die naturwissenschaftlichen Analysen wurden am Institut für Konservierungswissenschaften und Restaurierung der Universität für angewandte Kunst Wien mit finanzieller Unterstützung der Hochschuljubiläumsstiftung der Stadt Wien durchgeführt.

Lengyelkeramik wird nach dem Eponym-Fundort Lengyel in Südwestungarn genannt, der am Ende des 19. Jahrhunderts von M. Wosinsky (1888) entdeckt und ausgegraben wurde. Fundstellen mit derartiger Keramik sind, vor allem im heutigen Südmähren, Westslowakei, Westungarn und
Niederösterreich, häufig. Nach der ${ }^{14} \mathrm{C}$-Methode wird sie heute zwischen 4800 und 4200 a cal. BC datiert. Die Lengyelkeramik von Michelstetten weist nicht nur anhand ihrer Eigenschaften, sondern auch aufgrund ihrer absoluten Datierung, jüngere Züge auf. Charakteristisch für die Lengyelkeramik ist die auf die Gefäßoberflächen nach dem Brennen aufgetragene Bemalung, auch Kaltbemalung genannt. Die am häufigsten verwendeten Farben sind rot, weiß und gelb, die monochrom oder polychrom, oft kombiniert mit Knubben und/oder Ritzverzierungen, auf die äußeren und inneren Keramikoberflächen aufgetragen wurden. Darüber hinaus macht das häufig feine Aussehen ihrer keramischen Masse und unverwechselbaren Formen, das Interesse an ihrer Erforschung, insbesondere in der Kernregion, seit Ende des 19. Jahrhunderts, aus. Dennoch sind Untersuchungen $\mathrm{zu}$ den Herstellungstechniken von Lengyelkeramik mit Hilfe naturwissenschaftlicher Verfahren bis heute doch selten geblieben. Der jetzige Beitrag stellt selbst die ersten Ergebnisse derartiger Analysen, durchgeführt an Lengyelkeramik der Siedlung von Michelstetten, dar.

\section{Geografie und Geologie der Fundstelle}

In der Abbildung 1 ist der Raum um Michelstetten dargestellt. Geologisch gehört er zur Waschbergzone, die sich nördlich von Stockerau bis nördlich von Drasenhofen in SW-NO Richtung ausdehnt und das westliche vom östlichen Weinviertel trennt. In der leicht welligen Landschaft ragen 


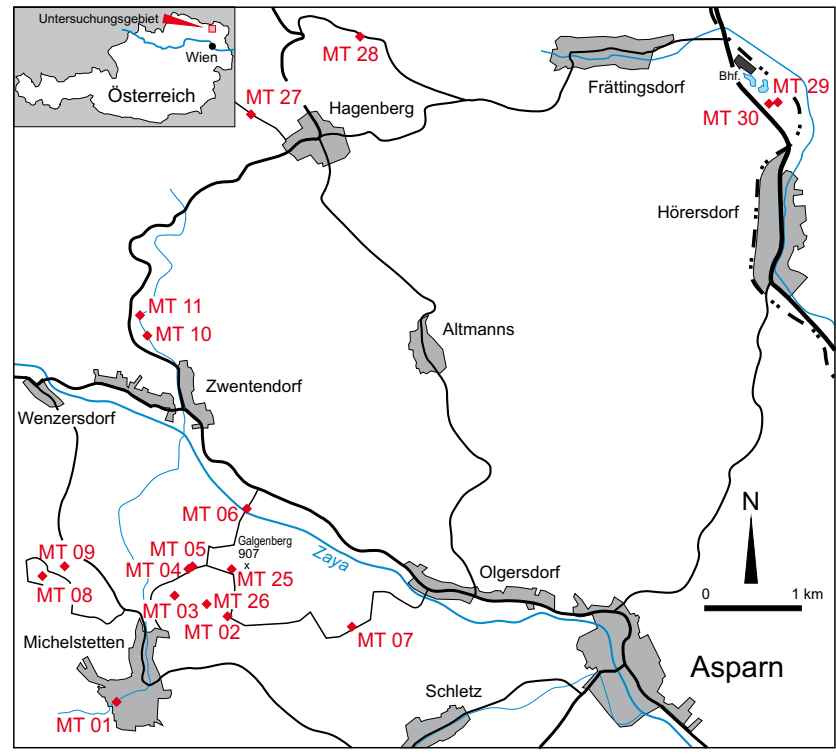

Abb. 1: Lage der prähistorischen Fundstelle von Michelstetten (roter Punkt) und der Umgebung mit Kennzeichnung der Stellen, in denen die Rohstoffproben entnommen wurden. Ausschnitt, umgezeichnet aus der Österreichischen Karte, Blatt 24 (Mistelbach), Maßstab 1: 50 000. (๑ BEV 2009, T2009/56720)

Fig. 1: Location (red dot) of the prehistorical find spot of Michelstetten and the surroundings with the places, where raw material samples were extracted (detailed map based on the Austrian Map, sheet 24 (Mistelbach), scale 1: 50 000. (๑ BEV 2009, T2009/56720)

diese Kalkberge hervor, die daher als Klippenzone bezeichnet werden. Sie wurden während der tertiären Bildung der Waschbergzone vom Untergrund abgetrennt und hochgeschürft. Die Leiser Berge, wo die prähistorische Fundstelle von Michelstetten an ihrem nördlichsten Ausläufer liegt, sind eine dieser Erhebungen. Wie GrILL (1968: 29-30) erfasst hat, besteht die Waschbergzone aus Jura- und Kreideablagerungen des Mesozoikums, die sandige Sedimente, Tonmergel, Tegel und lokal verbreitete Schotter, Konglomerate und Kalke in abwechselnder Abfolge enthalten. Ein reiches Spektrum maritimer Mikro- und Makrofossilien lässt ihren marinen Ursprung erkennen. Die Klentnitzer Schichten, welche großteils die Leiser Berge bilden, schließen eine vielfältige fossile Meeresfauna, wie Muscheln, Ammoniten, Belemniten und Foraminiferen ein (Thenius 1983: 42). Ebenfalls mariner Herkunft sind die weichen Sedimente der Michelstettener Formation, die dem oberen Oligozän und dem unteren Miozän zuzuordnen sind und auch viele Mikrofossilien enthalten (WESSELY 2006: 75). Meeresmikrofossilien finden sich zum Teil in der behandelten Lengyelkeramik wieder.

\section{Ausgangssituation der archäometrischen Untersuchungen}

Die Auswahl der archäometrisch untersuchten Keramikproben ergab sich aus der Auswahl von 2-3 Gefäßfragmenten jeweils aus einer der 15 Materialtypen, die aus der vorher vorgenommenen archäologischen Bestimmung des Keramikfabrikats mit Hilfe einer Lupe bei 8-facher Vergrößerung resultieren. Als Kriterien für die archäologische Beschreibung der Materialgruppen wurden Art und Aussehen, sowie die Qualität der Bearbeitung der plastischen Masse, das Verhältnis zwischen Menge und Größe der mineralischen Einschlüs- se, sowie ihre Verteilung, ihre Form und die Eigenschaft, die die einzelnen Materialgruppen am stärksten kennzeichnet, berücksichtigt (CARNEIRO 2002: 23-27).

Die Vielfalt der Korngröße der Tongruppen und ihre differenzierte Quantität führte zur Berücksichtigung aller vorhandenen Einschlussgrößen mit ihren Häufigkeiten, anstatt die häufigste Größe der Gesteine, wie es in der Archäologie üblich ist, aufzunehmen. Beispielsweise besitzt die Materialgruppe 1 pro $\mathrm{cm}^{2}$ gleichzeitig über 20 Partikel, die kleiner als $0,1 \mathrm{~mm}$ sind, 10 bis 20 Partikel, die von 0,1 bis $0,5 \mathrm{~mm}$ groß sind und weniger als 5 Einschlüsse, die zwischen 0,5 und 1,0 $\mathrm{mm}$ groß sind.

Weiters hat sich während der Aufnahme der Materialgruppen bald erwiesen, dass eine Bestimmung der Partikel unnötig war, weil erstens die erkennbaren Einschlüsse Quarz, Quarzit, Feldspat und Glimmer - in allen Tongruppen vorhanden sind und zweitens die Inklusionen, die Rückschlüsse auf die Tonzusammensetzung zulassen, genauer mittels Dünnschliffanalysen erfassbar sind. Deswegen wurden sie nicht ausführlich beschrieben, aber vermerkt, wenn sie in besonderen Verhältnissen zu bestimmten Materialgruppen standen.

Die Merkmale der Materialgruppen werden ausführlich in der Tabelle 1 dargestellt.

Hier wird nur kurz auf sie eingegangen. Die Grundmasse zur Keramikherstellung fast aller Materialgruppen hat ein feines lehmiges Aussehen, auch wenn sie mit vielen und großen Einschlüssen durchsetzt ist. Die 15 Materialtypen unterscheiden sich voneinander am deutlichsten durch die Größe und die Menge ihrer Partikeln. Mit der Ausnahme der Materialgruppen 1, 4 und 5 fallen alle anderen in den Bereich der grobkörnigen Materialgruppen, wobei einige weniger grob $(2,3,6,8,11,12,13)$ als andere $(7,9,10,14,15)$ sind. Außerdem besitzen einige Materialgruppen entweder mehr Glimmer $(4,11)$ oder Sand $(7,10,14,15)$ als die übrigen. Eine andere verbreitete Eigenschaft, die in allen Tongruppen beobachtet wurde, ist das Vorhandensein von Einschlüssen wie Kalk, Eisenoxyden, Meeresmikrofossilien und Tonbällchen. Manchmal treten mehrere Arten dieser Partikeln in einem Gefäß auf.

\section{Ergebnisse der archäometrischen Analysen}

\subsection{Die Keramikproben}

Die Keramikproben der naturwissenschaftlichen Analysen wurden als Mi01/01 bis Mi37/01 nummeriert und werden im Textteil als Mi01, 2, 3 ... simplifiziert erwähnt.

Nach den petrographischen und schwermineralischen Untersuchungen wurden die prähistorischen Keramikproben aufgrund der Unterschiede in der texturellen und mineralogischen Zusammensetzung in 20 unterschiedliche Keramiktypen von A bis T und im Brenngrad in 5 Varianten (Bh, C1, D1, Eh, F1) eingeteilt (Abb. 2-4).

Die Korrelation der Gruppierung A-T mit der Nummerierung der Keramikproben ist, wie folgt: A: Mi01, Mi30; B: Mi02, Mi03, Mi15; Bh: Mi06; C: Mi34; C1: Mi05; D: Mi19, Mi33; D1: Mi18; E: Mi26, Mi29; Eh: Mi07, Mi14; F: Mi10, Mi21, Mi23-Mi25, Mi35; F1: Mi11; G: Mi17, Mi22; H: Mi20; I: Mi36; J: Mi04; K: Mi16; L: Mi28; M: Mi31; N: Mi37; O: Mi32; P: Mi27; Q: Mi12; R: Mi09; S: Mi13; T: Mi08. 
Tabelle 1: Eigenschaften der Materialgruppen der Lengyelkeramik von Michelstetten.

Table 1: Properties of the material groups of the Lengyel ceramic from Michelstetten.

\begin{tabular}{|c|c|c|c|c|c|c|}
\hline $\begin{array}{l}\text { Material- } \\
\text { gruppen }\end{array}$ & $\begin{array}{l}\text { Art / Aussehen } \\
\text { der plastischen } \\
\text { Elemente der } \\
\text { keramischen } \\
\text { Masse }\end{array}$ & $\begin{array}{l}\text { Bearbeitung } \\
\text { der plastischen } \\
\text { Elemente der } \\
\text { keramischen } \\
\text { Masse }\end{array}$ & $\begin{array}{c}\text { Größe (mm) } \\
\text { und } \\
\left.\text { Menge (pro } \mathrm{cm}^{2}\right) \\
\text { der Einschlüsse }\end{array}$ & $\begin{array}{c}\text { Verteilung } \\
\text { der } \\
\text { Einschlüsse }\end{array}$ & $\begin{array}{c}\text { Form der } \\
\text { Einschlüsse }\end{array}$ & $\begin{array}{l}\text { Besondere } \\
\text { Merkmale }\end{array}$ \\
\hline 1 & $\begin{array}{c}\text { lehmartig, sehr fein, } \\
\text { dicht, } \\
\text { homogen, } \\
\text { wie geschlämmt }\end{array}$ & gut & $\begin{array}{c}<0,1 \mathrm{zu}>20 \\
0,1-0,5 \mathrm{zu} 10-20 \\
0,5-1,0 \mathrm{zu}<5\end{array}$ & gleichmäßig & $\begin{array}{l}\text { gerundet } \\
\text { und } \\
\text { kantig }\end{array}$ & \\
\hline 2 & $\begin{array}{l}\text { lehmartig, fein, } \\
\text { etwas locker, } \\
\text { homogen }\end{array}$ & gut & $\begin{array}{c}<0,1 \text { zu } 10-20 \\
0,1-1,0 \text { zu } 5-10 \\
1,0-2,0 \text { zu }<5\end{array}$ & ungleichmäßig & $\begin{array}{l}\text { gerundet } \\
\text { und } \\
\text { kantig }\end{array}$ & \\
\hline 3 & $\begin{array}{l}\text { lehmartig, fein, } \\
\text { etwas locker, } \\
\text { homogen }\end{array}$ & \pm gut & $\begin{array}{c}<0,1 \text { zu } 10-20 \\
0,1-1,0 \text { zu }>20 \\
1,0-2,0 \text { zu } 5-10 \\
>2,0 \text { zu }<5\end{array}$ & \pm gleichmäßig & $\begin{array}{l}\text { gerundet } \\
\text { und } \\
\text { kantig }\end{array}$ & \\
\hline 4 & $\begin{array}{c}\text { lehmartig, sehr fein, } \\
\text { dicht, } \\
\text { homogen, } \\
\text { wie geschlämmt }\end{array}$ & sehr gut & $<0,1-0,5 \mathrm{zu}>20$ & gleichmäßig & $\begin{array}{l}\text { gerundet } \\
\text { und } \\
\text { kantig }\end{array}$ & $\begin{array}{l}\text { viel und klein, } \\
\text { Glimmer }\end{array}$ \\
\hline 5 & $\begin{array}{c}\text { lehmartig, sehr fein, } \\
\text { dicht, } \\
\text { homogen, } \\
\text { wie geschlämmt }\end{array}$ & gut & $\begin{array}{c}<0,1 \text { zu } 10-20 \\
0,1-0,5 \text { zu } 5-10\end{array}$ & gleichmäßig & gerundet & \\
\hline 6 & $\begin{array}{l}\text { lehmartig, fein, } \\
\text { sehr dicht, } \\
\text { homogen }\end{array}$ & gut & $\begin{array}{c}<0,1 \mathrm{zu}>20 \\
0,1-0,5 \mathrm{zu} 10-20 \\
0,5-1,0 \mathrm{zu}<5\end{array}$ & gleichmäßig & $\begin{array}{l}\text { gerundet } \\
\text { und } \\
\text { kantig }\end{array}$ & \\
\hline 7 & $\begin{array}{l}\text { lehmartig, grob, } \\
\text { etwas locker, } \\
\text { homogen }\end{array}$ & gut & $<0,1-1,0 z u>20$ & gleichmäßig & $\begin{array}{l}\text { gerundet } \\
\text { und } \\
\text { kantig }\end{array}$ & viel Sand \\
\hline 8 & $\begin{array}{l}\text { lehmartig, fein, } \\
\text { etwas locker, } \\
\text { homogen }\end{array}$ & \pm gut & $\begin{array}{c}<0,1 \text { zu }>20 \\
0,1-1,0 \text { zu } 5-10 \\
1,0-2,0 \text { zu }<5\end{array}$ & ungleichmäßig & $\begin{array}{l}\text { gerundet } \\
\text { und } \\
\text { kantig }\end{array}$ & $\begin{array}{c}\text { etwas } \\
\text { pflanzliches } \\
\text { Material }\end{array}$ \\
\hline 9 & $\begin{array}{l}\text { lehmartig, fein, } \\
\text { etwas locker, } \\
\text { homogen }\end{array}$ & \pm gut & $\begin{array}{l}<0,1-2,0 \text { zu }>20 \\
2,0->3,0 \text { zu 5-10 }\end{array}$ & \pm gleichmäßig & gerundet & \\
\hline 10 & $\begin{array}{l}\text { lehmartig, fein, } \\
\text { etwas locker, } \\
\text { homogen }\end{array}$ & gut & $\begin{array}{c}<0,1-1,0 \mathrm{zu}>20 \\
1,0-2,0 \mathrm{zu}<5\end{array}$ & gleichmäßig & $\begin{array}{c}\text { gerundet } \\
\text { und } \\
\text { kantig }\end{array}$ & sehr viel Sand \\
\hline 11 & $\begin{array}{l}\text { sandartig, sehr fein, } \\
\text { dicht, } \\
\text { homogen }\end{array}$ & \pm gut & $\begin{array}{c}<0,1 \text { zu }>20 \\
0,5-1,0 \text { zu } 5-10 \\
1,0-2,0 \text { zu }<5\end{array}$ & \pm gleichmäßig & $\begin{array}{c}\text { gerundet } \\
\text { und } \\
\text { kantig }\end{array}$ & $\begin{array}{c}\text { viel } \\
\text { klein und groß, } \\
\text { Glimmer }\end{array}$ \\
\hline 12 & $\begin{array}{l}\text { lehmartig, fein, } \\
\text { etwas locker, } \\
\text { homogen }\end{array}$ & schlecht & $\begin{array}{c}<0,1 \mathrm{zu}>20 \\
0,5-2,0 \mathrm{zu} 5-10\end{array}$ & ungleichmäßig & $\begin{array}{l}\text { gerundet } \\
\text { und } \\
\text { kantig }\end{array}$ & $\begin{array}{c}\text { einige } \\
\text { Eisenoxide und } \\
\text { viele Tonbällchen }\end{array}$ \\
\hline 13 & $\begin{array}{c}\text { lehmartig, sehr fein, } \\
\text { dicht, } \\
\text { homogen, } \\
\text { wie geschlämmt }\end{array}$ & \pm gut & $\begin{array}{l}0,1-0,5 \text { zu >20 } \\
0,5-2,0 \text { zu 5-10 }\end{array}$ & ungleichmäßig & gerundet & $\begin{array}{c}\text { etwas } \\
\text { pflanzliches } \\
\text { Material }\end{array}$ \\
\hline 14 & $\begin{array}{l}\text { lehmartig, sehr fein, } \\
\text { dicht, } \\
\text { homogen }\end{array}$ & gut & $\begin{array}{c}<0,1-1,0 \mathrm{zu}>20 \\
1,0-2,0 \mathrm{zu}<5\end{array}$ & sehr gleichmäßig & $\begin{array}{l}\text { gerundet } \\
\text { und } \\
\text { kantig }\end{array}$ & sehr viel Sand \\
\hline 15 & $\begin{array}{l}\text { lehmartig, fein, } \\
\text { etwas locker, } \\
\text { homogen }\end{array}$ & \pm gut & $\begin{array}{l}<0,1-2,0 \text { zu }>20 \\
2,0-3,0 \text { zu } 5-10\end{array}$ & \pm gleichmäßig & $\begin{array}{l}\text { gerundet } \\
\text { und } \\
\text { kantig }\end{array}$ & \\
\hline
\end{tabular}

Die Grundmasse fast aller Keramiktypen hat ein feines Aussehen (besonders Mi08, 17, 22, 27, 36, 37), wobei einige glimmerig (Mi01, 9, 11-13, 20, 28, 30-32, 37), andere kalkhaltig (Mi04, 7, 10, 11, 14, 18-26, 29, 33, 36, 37) oder kalkarm (Mi02, 3, 6, 15, 16, 28, 31) bis kalkfrei (Mi08, 9, 12, 13, 27, 32), andere noch karbonhaltig (Mi01, 30) sind.

Der durchschnittliche Anteil an nicht-plastischen Elementen ist unterschiedlich und kann in drei Kategorien gruppiert werden: zwischen 16-19 \% (Mi05, 7, 14, 17, 22, 26, 29, 34), zwischen 26-33\% (Mi04, 10-13, 18-21, 23-25, 28, 31-33) und zwischen 36-46 \% (Mi01-3, 6, 8, 9, 15, 16, 27, 30, 36, 37). Die mittlere Größe der nicht-plastischen Elemente, in natürlicher
Form in den Rohstoffen vorhanden, ist recht variabel und kann als etwas feiner, zwischen 0,03-0,05 mm (Mi02, 3, 6, 15, $16,18-20,27,33,36)$, weniger fein, zwischen $0,06-0,09 \mathrm{~mm}$ (Mi01, 4, 5, 7, 14, 17, 22, 26, 28, 29, 30, 34), grob, zwischen 0,10$0,17 \mathrm{~mm}$ (Mi08, 10, 11, 13, 21, 23-25, 31, 37) und sehr grob, zwischen 0,20-0,30 mm (Mi09, 12, 32) angesprochen werden. Sie bestehen hauptsächlich aus monokristallinen Quarzen und/oder Muskovit in unterschiedlicher Menge, manchmal untergeordnet auch noch aus Karbonaten (Mi01, 30), Alkalifeldspaten (Mi016) und oxidiertem Glaukonit (Mi04).

Die Keramik einiger Proben hat neben den nicht-plastischen Elementen, die in der Grundmasse natürlich vorkom- 
men können, auch noch gröbere Partikel, die andere mineralogische Zusammensetzung und schlechteren Abrundungsgrad aufweisen. Der mittlere Wert ihrer Korngröße verteilt sich in einer Skala von 0,7-2,4 mm (Mi01, 30), 0,8-3,16 mm (Mi02, 3, 6, 15), 0,83-2,36 mm (Mi16), 0,8-3,68 mm (Mi18, 19, 33), über 1-2,12 mm: (Mi27) bis 1,36-4,4 mm (Mi20). Dieser Teil der Keramik scheint künstlich, meist durch den Zusatz von grobem Sand (poly- und monokristallinen Quarzen), gemagert worden zu sein.

Anderer Teil der Keramik wurde noch mit Ton/Siltsteinklastern bzw. „Schamottbröckchen“ (Mi04, 10-12, 18, 19, 21, 23-25, 33) und/oder Pflanzenfasern (Mi01, 7, 10, 11, 14, 18, 19, 21, 23-26, 29, 30, 33) gemagert.

Das Vorhandensein von echtem Schamott scheint sich durch die teilweise sehr eckigen und scharfkantigen, siltigen Bröckchen auszuzeichnen, die gebrannter Keramik gleichen. Solch scharfkantige Brüche sind normalerweise bei Alttonbröckchen (eingetrocknete und wieder zugesetzte Tonbröckchen oder natürliche verfestigte Tonsteinklaster) nicht zu erwarten. Etwa $25 \%$ des Magerungsanteils der Keramikproben F (Mi10, 21, 23-25, 35) besteht aus solchen Partikeln, die bis 4,2 mm lang sein können. Bei Keramikprobe Mi11 ist ihr Anteil wesentlich reduziert (6\%), dennoch signifikant.

Die organischen Magerungsmaterialien bestanden ursprünglich (v. a. bei Keramikproben Mi07, 14, 26, 29) aus länglichen, feinen Pflanzenfasern, die im Dünnschliff eine bis zu 3,5 mm Länge erreichen konnten und jetzt als Pseudomorphosen in Form länglicher schmaler Poren vorliegen.

Die Sortierung der Körner ist in der Regel bimodal (Mi0107, 14-20, 22, 26-30, 33, 34, 36), wobei manche Keramikrohstoffe teilweise ziemlich inhomogen (schlecht aufbereitet) zu sein scheinen (Mi08-13, 21, 23-25, 31, 32, 35, 37). Solche Proben lassen sich nicht nur durch eine größere Probenserie voneinander eindeutig abgrenzen, sondern deuten ebenfalls auf wenig Sorgfalt in der Aufarbeitung der Keramikrohstoffe hin. Ein weiterer Nachweis schlechter Tonaufarbeitung können die nicht resorbierten, eingestreuten Tonbröckchen mancher Proben darstellen, die häufig eine hellere oder dunklere Farbe als die der Grundmasse zeigen (Mi01, 10, 11, 20, 21, 23-25, 30).

Die Matrix der Proben ist großteils kalkhaltig (siehe oben) bzw. sind Kalkeinschlüsse teilweise mit bloßem Auge noch sichtbar. Das Vorhandensein dieses Calciumkarbonats deutet auf Brenntemperaturen der Keramik unter $800^{\circ} \mathrm{C}$ hin, da sich ab solchen Brenntemperaturen die Calciumkarbonate zersetzen und daher nicht mehr direkt bestimmbar sind. Darüber hinaus berichtet HENNICKE (1989: 17), dass unabhängig von der Ofenart, Keramik mit Brandtemperaturen ab $600^{\circ} \mathrm{C}$ erhaltbar und witterungsbeständig ist. Infolgedessen konnte die Keramik von Michelstetten bei 600 und unter $800^{\circ} \mathrm{C}$ gebrannt worden sein.
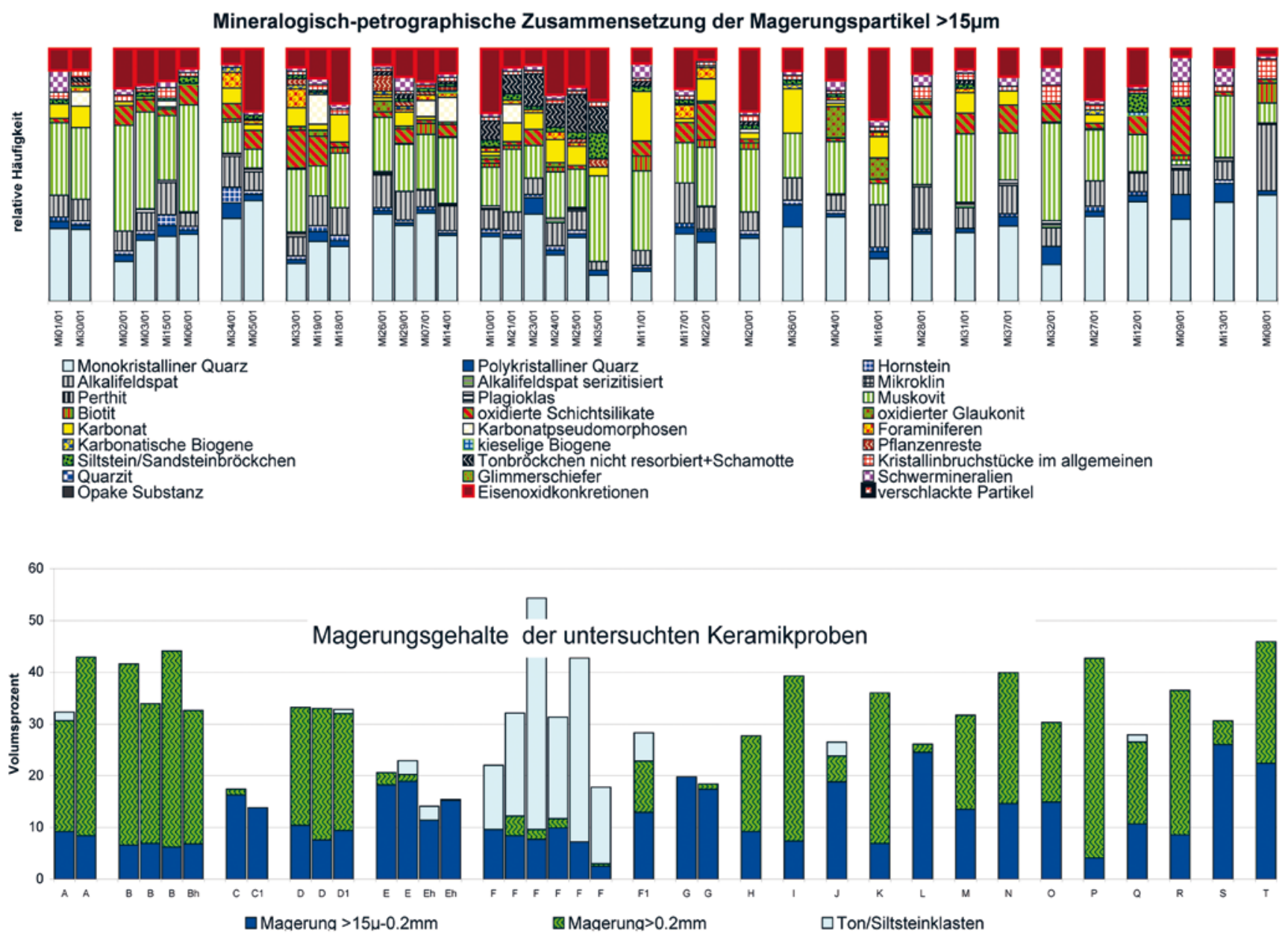

Abb. 2: Mineralogisch-petrographische Zusammensetzung der Magerungspartikel der Keramikproben (>15 $\mu \mathrm{m})$.

Fig. 2: Mineralogic-petrographic composition of temper particles of the ceramic samples (>15 $\mu \mathrm{m})$. 

(ohne Schamottepartikel)

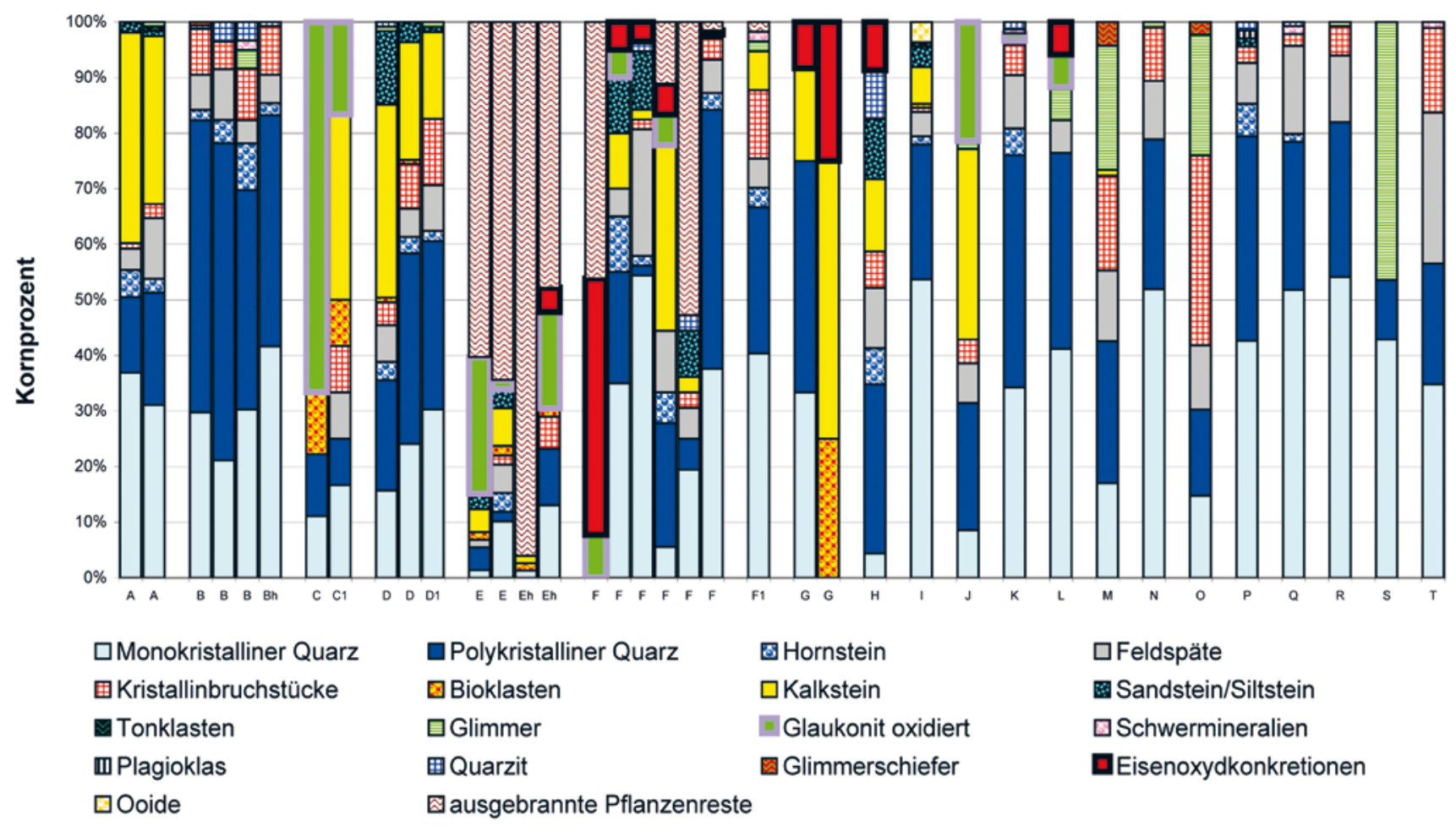

Abb. 3: Mineralogisch-petrographische Zusammensetzung der groben Magerungspartikel (>2,0 mm) der Keramikproben ohne Schamottepartikel. Fig. 3: Mineralogic-petrographic composition of coarse temper particles (>2.0 mm) of the ceramic samples (> $2.0 \mathrm{~mm})$ without fireclay particles.

In fast allen Keramikproben treten Eisenoxidkonkretionen in der Grundmasse häufig (Mi02-07, 10-12, 14-29, 3337) oder untergeordnet (Mi08, 09, 13, 31, 32) auf, was für eine allgemeine Verwendung eisenhaltiger Rohstoffe spricht. Andererseits deuten Beobachtungen der Keramik von Michelstetten - wie abwechselnde Farbe der Keramikoberflächen von rot über beige und braun bis grau und schwarz und die Farbübergänge des Scherbenbruches von rot zu orange oder braun von den Gefäßoberflächen in Richtung Kern, meist mit braunen bzw. grauen Kernfarben - auf schwache und unregelmäßige Brenntemperaturen hin. Solche Farbnuancen entstehen am häufigsten beim Keramikbrennen unter Verwendung offener Brennanlagen, wie einfacher Meiler und Feldöfen. Bei offenen Brennanlagen besteht die Gasatmosphäre während des Brandes aus einer Mischung von Luft und Gasen, die bei der Verbrennung des Heizmaterials unter abwechselnder Windrichtung entsteht. Dadurch kann sich die oxidierende Brennatmosphäre nicht lang halten, bzw. wechselt die oxidierende und reduzierende Atmosphäre ab, wie es schon Noll (1991: 86) in seinen Keramikbrennexperimenten merkte. Unter solchen Brennbedienungen neigt die reduzierende Atmosphäre zu überwiegen und die Keramik zeigt häufig dunklere Farbtöne. Da die Oberflächen der Keramik von Michelstetten im Allgemeinen oxidierende Brennfarben (rot, orange, braun, beige) bei dunkleren Kernen aufweisen, konnte die rote Färbung ihrer Oberflächen erst in der Endphase des Brennvorganges entstanden sein, die anschließend durch das häufige Vorhandensein eisenhaltigen Herstellungsmaterials, die die rote Farbe betonen, verstärkt werden konnte. Tatsächlich ist es bekannt, dass die Farbe des Eisens sich schon bei Temperaturen ab $250^{\circ} \mathrm{C}$ und in einem schwach oxidierenden Milieu ändern kann: W. NolL (1991: 90). Andererseits zeigen Keramikbrennexperimente in offenen Anlagen, dass Sauerstoff erst nach der Verbrennung des Brennmaterials, während der Abkühlungsphase, dann in einer gasfreien Brennatmosphäre, die Keramik erreichen kann (Noll 1991: 88). Unter Verwendung offener Brennanlagen bei der Herstellung der Keramik von Michelstetten wären die Brenntemperaturen der oxidierenden Endphase noch hoch genug, damit chemische Verbindungen mit den Eisenoxiden an den Oberflächen entstanden, wodurch sie, und nicht mehr ihr Kern, rot gefärbt würden.

\subsection{Die Rohstoffproben}

Zur Ergänzung der wissenschaftlichen Untersuchungen und zur Charakterisierung lokal verfügbarer Rohstoffe wurden 22 Rohstoffproben aus einem Bereich von etwa $100 \mathrm{~km}^{2}$ (Lat. $21^{\circ} 60^{\prime} 00^{\prime} \mathrm{N}$ bis $28^{\circ} 00^{\prime} 00^{\prime} \mathrm{N}$, Long. $34^{\circ} 00^{\prime} 00^{\prime} \mathrm{E}$ bis $38^{\circ} 00^{\prime} 00^{\prime}$ E) entnommen, wobei die meisten aus der näheren Umgebung von Michelstetten stammen (Abb. 1). Sie wurden als MT01-MT11 und MT20-MT30 kennzeichnet (Abb. 5) und im nachfolgenden Text als Rohstoffproben MT01, 02, 03 ... bezeichnet.

In Michelstetten kommen auf engstem Raum zahlreiche Formationen unterschiedlichen geologischen Alters vor (Quartär bis Oberjura), die zusätzlich noch teilweise tektonisch verschuppt worden sind und als Rohstofflieferanten in Frage kommen. Es wurde versucht, möglichst von allen geologischen Formationen, die dort als Rohstoffquellen in Frage kommen, Proben zu bekommen.

Die Lehm- und Tonproben wurden nach Absieben der 


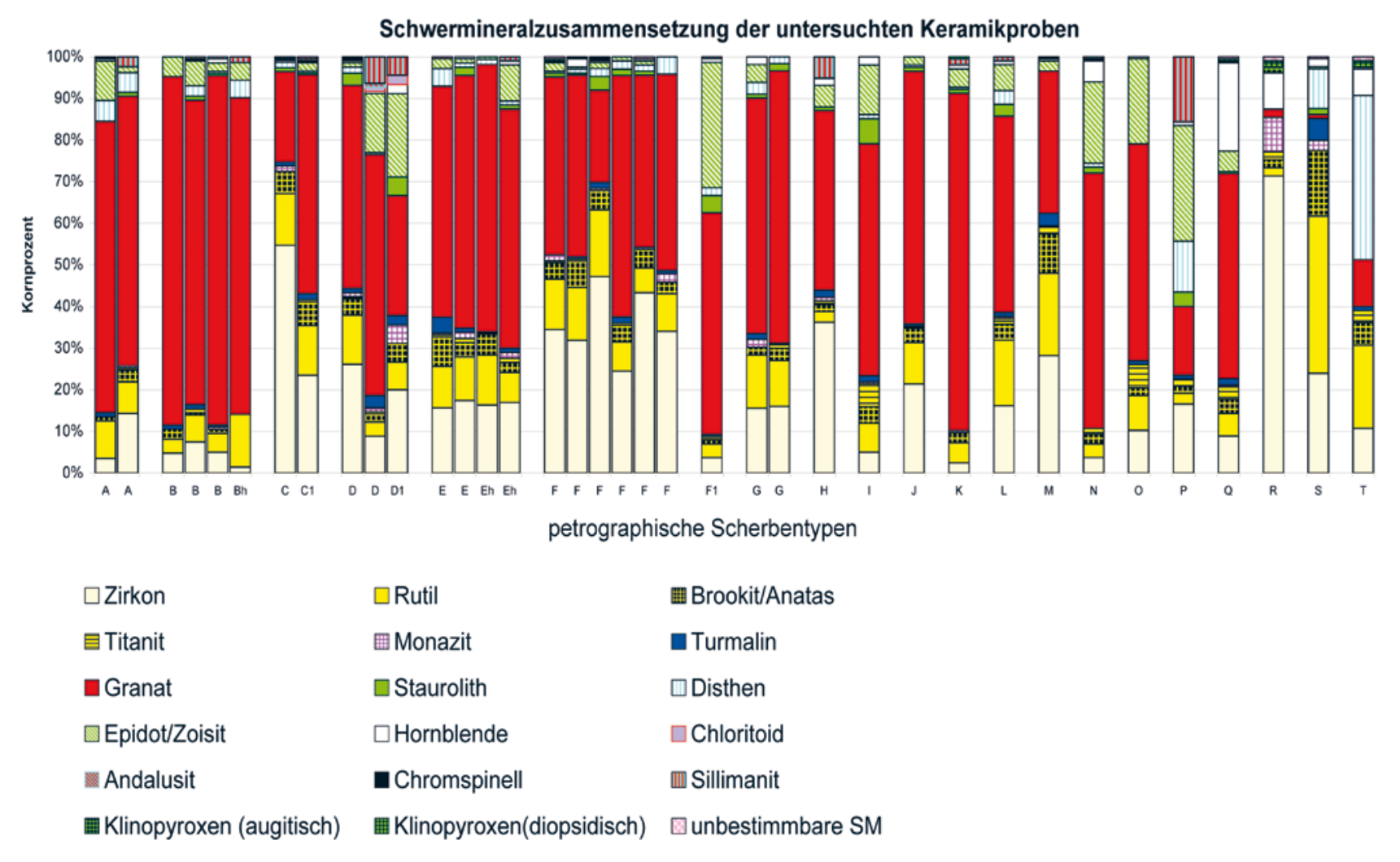

Abb. 4: Schwermineralzusammensetzung der Keramikproben.

Fig. 4: Heavy mineral composition of ceramic samples.

Partikel gröber als $2 \mathrm{~mm}$ im Labor zu kleinen Ziegelchen geformt und bei $750^{\circ} \mathrm{C}$ gebrannt. Diese Ziegel wurden dann gleich wie die Keramik untersucht - Dünnschliff und bei genügender Probemenge $(>5 \mathrm{~g})$ auch Schwermineralanalysen (Abb. 5). Während die Tonrohstoffe und Lehmvorkommen relativ vollständig untersucht werden konnten, sind Proben gröberer Sande, die als künstliches Magerungsmaterial in einiger Keramik vorkommen, möglicherweise nicht ausreichend berücksichtigt worden.

Aufgrund der teilweise schlechten Aufschlussverhältnisse konnten häufig nur sehr oberflächennahe und verunreinigte Proben entnommen werden. Diese Proben stellen daher häufig „Mischproben“ mehrerer geologischer Formationen dar. Eine klare stratigraphische Ansprache dieser Proben ist dann schwierig. Gerade diese Proben eignen sich häufig als Vergleich bzw. entsprechen sie manchmal sogar direkt den verwendeten Keramikrohstoffen. Das lässt darauf schließen, dass die Töpfer der Lengyelkeramik aus Michelstetten oft oberflächennahe Rohstoffe und infolgedessen mit weniger Aufwand ausgesucht haben.

Proben folgender Formationen wurden für Vergleichszwecke untersucht und nach der aktuellen Nomenklatur bzw. nach der Nummerierung der Geologischen Karte für Niederösterreich 2002 parallelisiert. Diese steht in Klammern am Ende der Anführung einzelner geologischen Schichten (Schnabel, Krenmayr \& Roetzel 2002: 5-6, 20-30; WesseLY 2006: 41-75).

MT01, 06, 20, 21, 29, 30: Quartäre Lösslehme von allgemeinen Ausschneidungen (19);

MT07: sandiger Lehm der Hollabrunn-Mistelbach-Formati- on des Pannoniums aus dem oberen Miozän (111, Molassezone)

MT22-24: Marine Tontegel des Badeniums aus dem mittleren Miozän (113, Molassezone)

MT10, 11, 27, 28: Schieferige Tonmergel der Zdanice/Hustopece-Formation, von Eggenburgium bis Ottnangium, aus dem unteren Miozän (147, östl. Teil der Waschbergzone)

MT08, 09: Marine Tone bis sandige Mergel der Michelstetten-Formation des Egeriums aus dem oberen Oligozän und dem unteren Miozän, vermutlich mit Pannonsand oder Löss vermischt (149, Außenrand der Waschbergzone)

MT04, 26: Mergel, Mergelkalke und Oolithe der KlentnitzerFormation des Oxfordiums und des Tithoniums aus dem Senon (158, Waschbergzone)

MT02, 03: Umgelagerte, zum Teil verunreinigte Kreidesedimente aus dem Senon und dem Paläozän (genauere geologische Zuordnung nicht möglich)

MT05: Tegel aus dem Senon (genauere geologische Zuordnung nicht möglich)

MT25: Oberflächenmischprobe des Senons und des Quartärs (genauere geologische Zuordnung nicht möglich).

\subsection{Herkunftsmäßig interpretierte Keramikproben}

Nach den naturwissenschaftlichen Untersuchungen scheint ein Großteil der beprobten prähistorischen Keramik mit Rohstoffen, die in der Nähe der Fundstelle entnommen wurden, hergestellt worden sein. Generell kann gesagt werden, dass praktisch sämtliche, lokal vorkommende Rohstoffe, wie quartäre Lehme, jung- und alttertiäre bis oberkretazische Tonmergel, verwendet worden sind. Es ist auch Keramik 

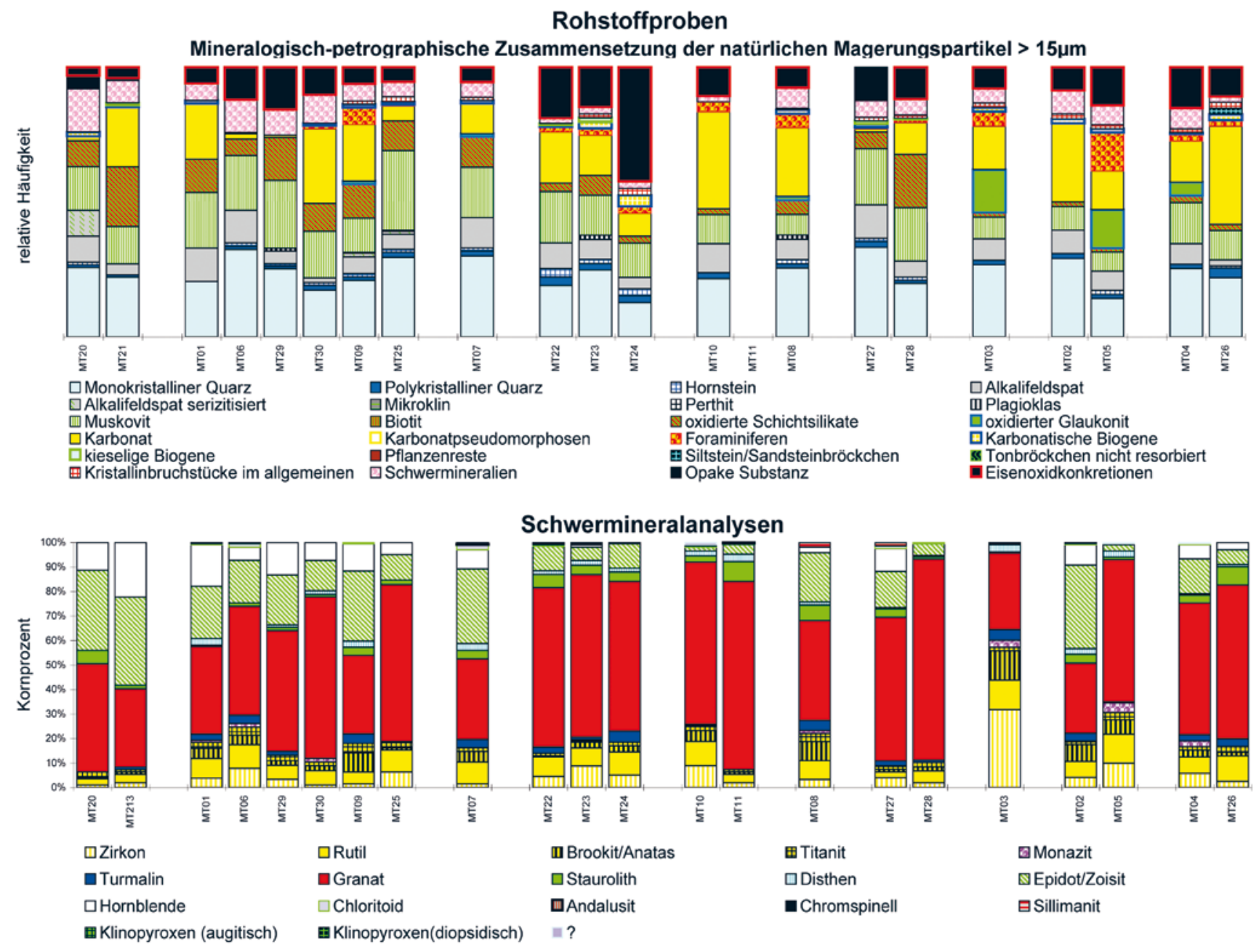

Abb. 5: Mineralogisch-petrographische Zusammensetzung und Schwermineralzusammensetzung der Magerungspartikel der Rohstoffproben (>15 $\mu$ m).

Fig. 5: Mineralogic-petrographic and heavy mineral composition of temper particles of raw material samples (>15 $\mu \mathrm{m})$.

vorhanden, die mit nicht lokalen Rohstoffen erzeugt wurde. Bei dieser wird ihr Ursprungsgebiet nur angedeutet, weil keine weiteren Untersuchungen zur Erschließung ihrer genaueren Herkunft gemacht worden sind. Das Material anderer Keramikproben konnte schwer mit den erfassten lokalen Rohstoffen in Verbindung gebracht werden. Bei diesen kann es sich um Keramik handeln, die entweder aus mehreren lokalen Materialien bestehen, also Mischproben darstellen, deren Herkunft nicht leicht nachgewiesen werden kann oder mit nicht-lokalen Rohstoffen hergestellt wurden.

Keramikproben, die die geologischen Formationen der Umgebung der prähistorischen Fundstelle von Michelstetten entsprechen, sind:

Mi02, 03, 06, 15: entkalkte Tone aus dem Schieferigen Tonmergel (Zdanice-Hustopece-Formation) oder des Pannon.

Mi04, 05, 07, 10, 14, 18, 19, 20?, 21, 23-26, 29, 33-35: senone Tonmergel.

Mi28: wahrscheinlich entkalkter, lokaler, (quartärer?) Rohstoff oder Senonmergel.

Mi11: vermutlich ein etwas anders zusammengesetzter Tonmergel, der möglicherweise durch quartäres Material verunreinigt worden ist.

Mi16: mariner Tonmergel des Senon.

Mi31: stark sandiger, tertiärer Tonmergel mit marinen Mikrofossilien.
Mi17, 22: mariner, neogener oder senoner, nicht künstlich gemagerter Tonmergel.

Mi37: quartärer, lokaler Lehm.

Folgende Probe lässt sich derzeit am ehesten mit der geologischen Formation eines von Michelstetten etwas entfernten Gebiets vergleichen:

Mi36: mariner Tonmergel, gemagert mit einem flachmarinen Sand bzw. Kalksandstein (Kalkooide).

Kalkooide kommen besonders häufig in Flachwasserablagerungen des Sarmat vor, wie laut geologischer Karte im nächstgelegenen Vorkommen von Sarmatsedimenten bei Hauskirchen, das ca. $24 \mathrm{~km}$ NO von Michelstetten entfernt liegt. Möglicherweise können solche Sedimente aber auch in anderen Ablagerungen auftreten. In den aufgesammelten Rohstoffen in der Umgebung von Michelstetten konnten solche oxidführenden Sande jedenfalls bisher nicht gefunden werden.

Keramikproben, deren Rohstoffherkunft derzeit ungewiss bleibt, sind:

Mi01,30: ein unmittelbar lokal anstehender Rohstoff mit derartig gut gerundeten Karbonatpartikeln ist derzeit lokal nicht bekannt. Es ist theoretisch auch möglich, dass die Proben mit Tonmergel marinen Ursprungs (Foraminiferen) und Sanden der schieferigen Tonmergel erzeugt worden sind. In diesem 
Fall würde es sie sich um Mischproben aus lokalen Rohstoffen handeln.

Mi12: relativ untypisch ausgebildet, hauptsächlich durch relativ hohen Hornblendegehalt gekennzeichnet. Es liegt nahe, dass diese Hornblendekörner mit dem sandigen Magerungsmaterial in die Proben gekommen sind. Hornblende ist eher typisch für ganz junge Sedimente, quartäre Lehme oder Verwitterungslehme. Eine weitere Interpretation ist aber bei dieser Einzelprobe derzeit nicht möglich.

Mi27: ist im Dünnschliff den Proben Mi02, 03, 06 und 15 sehr ähnlich, aber mit einer völlig unterschiedlichen Schwermineralzusammensetzung, wobei nur eine relativ geringe Anzahl von Schwermineralien untersucht werden konnte. Das Vorhandensein von Epidotvormacht mit reichlich Sillimanit und Disthen lässt diese Einzelprobe ohne vergleichbare Herkunft der Rohstoffe erscheinen.

Eine lokale Herkunft der verwendeten Rohstoffe folgender Keramikproben ist aufgrund der schwermineralogischen Zusammensetzung auszuschließen. Leider lassen sich mögliche Herkunfsorte der nicht lokalen Keramikproben mangels an Vergleichsproben derzeit nicht näher eingrenzen.

Mi08: ist im Dünnschliff hauptsächlich durch den hohen Gehalt an feinkörnigen Feldspatkörnern, sowie an sehr charakteristischen Gesteinsbruchstückchen und Quarz-Perthitaggregaten gekennzeichnet. Das Schwermineralspektrum weicht durch die Disthen- und Rutilvormachten völlig von den lokal bekannten Rohstoffen ab. Vermutlich wurde die Probe, wie auch Probe Mi09, aus Verwitterungslehmen, wie sie in Kristallingebieten der Böhmischen Masse über hochmetamorphen Kristallingesteinen (z.B. Granulit) vorkommen können, erzeugt.

Mi09: ist im Dünnschliff durch einen auffällig erhöhten Gehalt an dunklen, z. T. oxidierten Glimmern (Biotit) gekennzeichnet. Das Schwermineralspektrum zeichnet sich durch massive Zirkonvormachten aus. Auffällig ist auch noch das Auftreten von Monazit, Hornblende und Klinopyroxen.

Mi13: ist im Dünnschliff hauptsächlich durch den hohen Glimmergehalt gekennzeichnet. Vermutlich wurde sie aus Verwitterungslehmen, wie sie in Kristallingebieten der Böhmischen Masse vorkommen können, erzeugt.

Mi32: hat eine Magerung mit einem extrem hohen Anteil an Kristallinbruchstücken (Glimmerschiefer und Gneisfragmente). Lokal konnten solche Rohstoffe bisher aber nicht gefunden werden.

\subsection{Untersuchungen der Überzüge und Malmischungen}

$8.8 \%$ der Keramik aus Michelstetten weist Reste von Kaltbemalung (Abb. 6) auf.

Neben der Kaltbemalung wurde hochglänzend monochrome, rote oder schwarze polierte Keramik hergestellt, die aufgrund ihrer Ähnlichkeit mit der römischen Ware als „unechte Terra Sigillata“ und „unechte Terra Nigra“ Keramik bezeichnet wurde ( PAlliardi 1914: 9). Die schwarze hochpolierte Keramik (Abb. 7) könnte mit Hilfe reduzierender Brennverfahren, eventuell unter Verwendung zusätzlicher organischer Stoffe (wie Asche, Holzkohle und Tierfett) in der Keramikmasse erzeugt werden, wie es Experimente durch KovARNíK (1983-84: 166-167) anführten.

Die Untersuchungen der Keramik von Michelstetten belegen, dass es sich bei der roten, hochpolierten Keramik, um rote Überzüge handelt, wie sie im Querschnitt mancher Gefäße genauer zu beobachten sind (Abb. 8).

Die archäologische Analyse ihrer Oberflächen ergibt, dass sie sowohl in dünnem Anstrich, wie Malfarben, als auch in flüssigen bis dickeren Überzügen auf die fertigen, ungebrannten Gefäße aufgetragen wurden.

16 Analysen der Malfarben an 11 bemalten Gefäßen von Michelstetten wurden mittels Röntgendiffraktometrie und Polarisationsmikroskopie durchgeführt und als MIP 01 bis MIP 11 bezeichnet (Tab. 2).

Es handelt sich dabei um die mineralogische Zusammensetzung und die farbgebende Substanz der Malmischungen und Überzüge zu bestimmen. Die Ergebnisse der mineralogischen Farbzusammensetzung sind einheitlich:

Die weiße Farbe besteht immer aus zerriebenem, feinkörnigem und grobkörnigem Kalzit (MIP 01, 03, 04a, 06a, 8-10, 11a).

Die rote Farbe wurde aus verschiedenen Mischungen zusammengesetzt: eine Gemenge aus feinkörnigem Hämatit, Eisenoxiden, Quarz und Muskovit (MIP 02, 06b, 11b); eine Mischung aus feinkörnigen Eisenoxiden mit Quarz und Tonmineralien (MIP 04b); eine Vermengung aus feinkörnigen Eisenoxiden und Ton, verunreinigt mit Quarz (MIP 05b) oder aus Eisenoxiden mit Tonsubstanz (MIP 07b) vermischt.

Die gelbe Farbe setzt sich aus einer feinkörnigen Mischung von Kalkspatpulver, etwas Quarz und gelben Eisensulfaten zusammen (MIP 05a). Die geringe Probemenge der einzigen Analyse erlaubt keine schlüssigen Aussagen zum

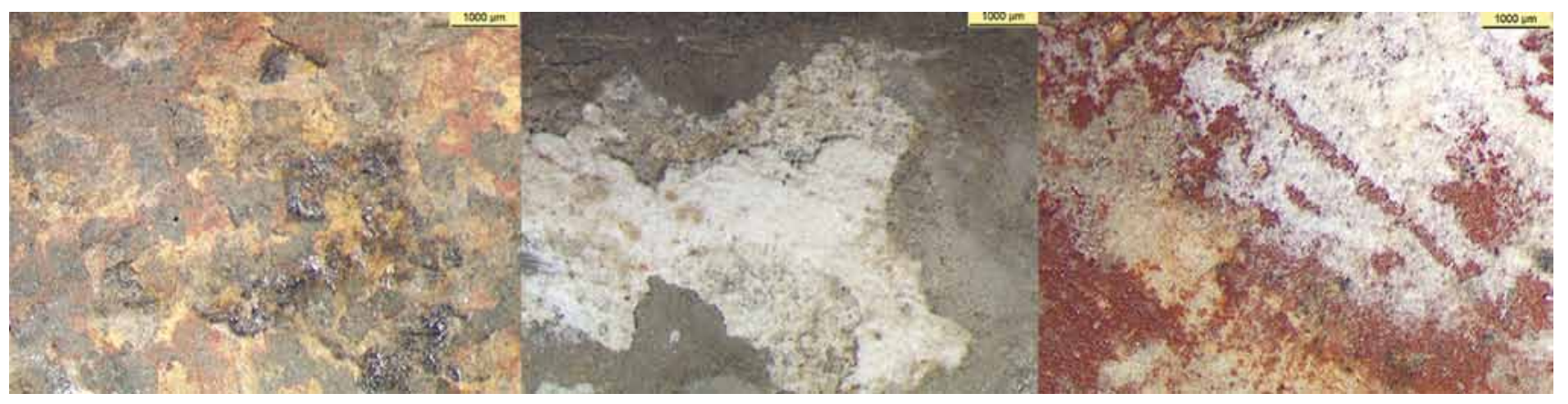

Abb. 6: Farbkombinationen der bemalten Keramik - gelbrot, weiß und rotweiß.

Fig. 6: Colour combinations of the painted ceramics - yellow red, white and red white. 


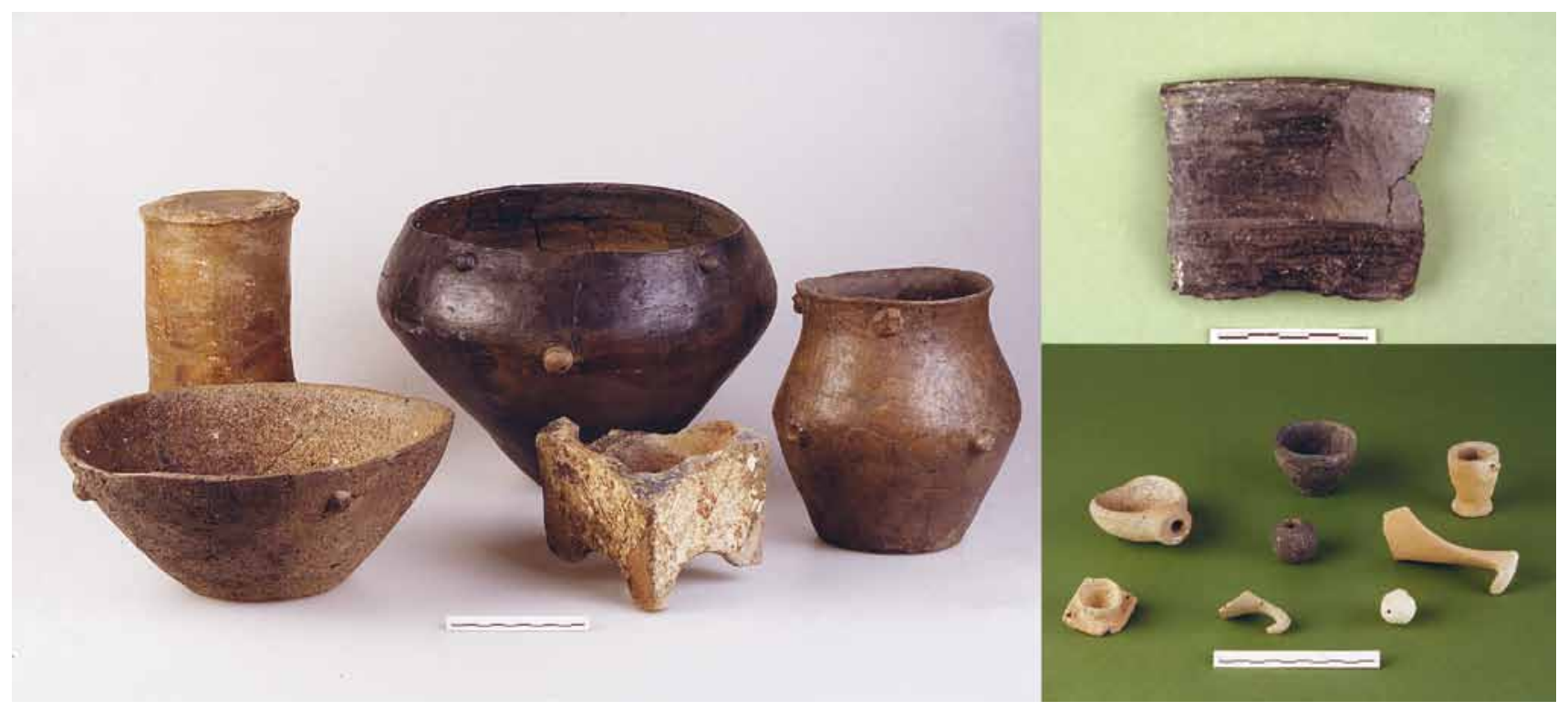

Abb. 7: Auswahl charakteristischer Lengyelkeramik mit einem Exemplar der „unechten Terra Nigra“ Keramik (rechts, oben).

Fig. 7: Selection of characteristic Lengyel ceramics with an exemplar of "artificial Terra Nigra” ceramic (top right).

Gehalt der gelben Farbe. Übrigens ist sie das einzige Beispiel gelber Malfarbe unter der Keramik von Michelstetten und für die Lengyelkeramik im Allgemeinen in diesem Zeitraum unüblich.

Weitere Ergebnisse zur Farbstoffauswahl der Lengyeltöpfer von Keramik anderer Funstellen erweitern etwas die bisherigen Kenntnisse. Die gelbe Pigmentierung der kalt bemalten Keramik der Lengyelkultur im südmährischen und ostösterreichischen Raum scheint vor allem während der Frühstufe (um etwa 4700-4600 vor Chr.) verwendet worden sein und allein auf dem Mineral Jarosit in reiner Form zurückzuführen sein (KovARNík 1989: 157; DonEus 2001: 44). Die weiße Farbe besteht in Kamegg (Niederösterreich) größtenteils aus Kaolinit (Doneus 2001: 44). In Mähren gibt es derzeit keine mineralogische Bestimmung des weißen Pigmentes. Es wird vermutet, dass es sich um Dolomit, Kaolin oder Kreide handeln könnte (KovarNík 1989: 151). Die rote Farbe scheint in Kamegg, Falkenstein (Niederösterreich) und Südmähren ausschließlich aus Hämatit zu bestehen (DRAžDÁK 1973-74: 79; Neugebauer-Maresch 1981: 86; Doneus 2001: 44), genauso wie das rote farbgebende Mineral der Keramik aus Michelstetten.

In Michelstetten ähnelt die chemische Zusammensetzung der roten Malfarben kaltbemalter Keramik dem Gemenge der roten Keramiküberzüge. Das heißt, sowohl die Malfarben der Kaltbemalung als auch die Mischung der Überzüge wurden nach demselben Rezept vorbereitet, wobei manche Überzüge nur etwas dicker waren. Überzüge sind meiner Auffassung nach als Bemalungstechnik anzusehen. Die Besonderheit der Überzüge in Bezug auf die herkömmliche Kaltbemalung ist, dass Überzüge vor dem Brand, und nicht nach dem Brand, auf die Gefäßoberflächen aufgetragen wurden und das Feuer
Tab. 2: Mineralogische Zusammensetzung der Malfarben und Überzüge bemalter Gefäße.

Table 2: Mineralogical composition of the colours and coatings on painted vessels.

\begin{tabular}{|l|c|l|l|}
\hline Prb.Nr. & Inv.Nr. & Farbe & \\
\hline MIP 01 & 683 & weiss & Mischung aus zerriebenem, feinkörnigem und grobkörnigem Kalzit \\
\hline MIP 02 & 1439 & rot & Gemenge aus feinkörnigem Hämatit, Eisenoxiden, Quarz und Muskovit \\
\hline MIP 03 & 1880 & weiss & Mischung aus zerriebenem, feinkörnigem und grobkörnigem Kalzit \\
\hline MIP 04a & 1880 & weiss & Mischung aus zerriebenem, feinkörnigem und grobkörnigem Kalzit \\
\hline MIP 04b & 2468 & rot & Feinkörnige Eisenoxide mit Quarz und Tonmineralen vermengt \\
\hline MIP 05a & 5847 & gelb & Gemenge aus feinkörnigem Kalzit, Eisen(hydro)xiden und Ton mit Quarz verunreinigt \\
\hline MIP 05b & 5847 & rot & Mischung aus feinkörnigen Eisenoxiden und Ton mit Quarz verunreinigt \\
\hline MIP 06a & 5768 & weiss & Gemenge aus zerriebenem, feinkörnigem und grobkörnigem Kalzit \\
\hline MIP 06b & 5768 & rot & Mischung aus feinkörnigem Hämatit, Eisenoxiden, Quarz und Muskovit \\
\hline MIP 07a & 4676 & weiss & Mischung aus feinkörnigem, zerriebenem und grobkörnigem Kalzit \\
\hline MIP 07b & 4676 & rot & Mischung aus Eisenoxiden mit Tonsubstanz \\
\hline MIP 08 & 4834 & weiss & Gemenge aus zerriebenem, feinkörnigem und grobkörnigem Kalzit \\
\hline MIP 09 & 4831 & weiss & Gemenge aus zerriebenem, feinkörnigem und grobkörnigem Kalzit \\
\hline MIP 10 & 4722 & weiss & Gemenge aus zerriebenem, feinkörnigem und grobkörnigem Kalzit \\
\hline MIP 11a & 5378 & weiss & Mischung aus zerriebenem, feinkörnigem und grobkörnigem Kalzit \\
\hline MIP 11b & 5378 & rot & Mischung aus feinkörnigem Hämatit, Eisenoxiden, Quarz und Muskovit \\
\hline
\end{tabular}




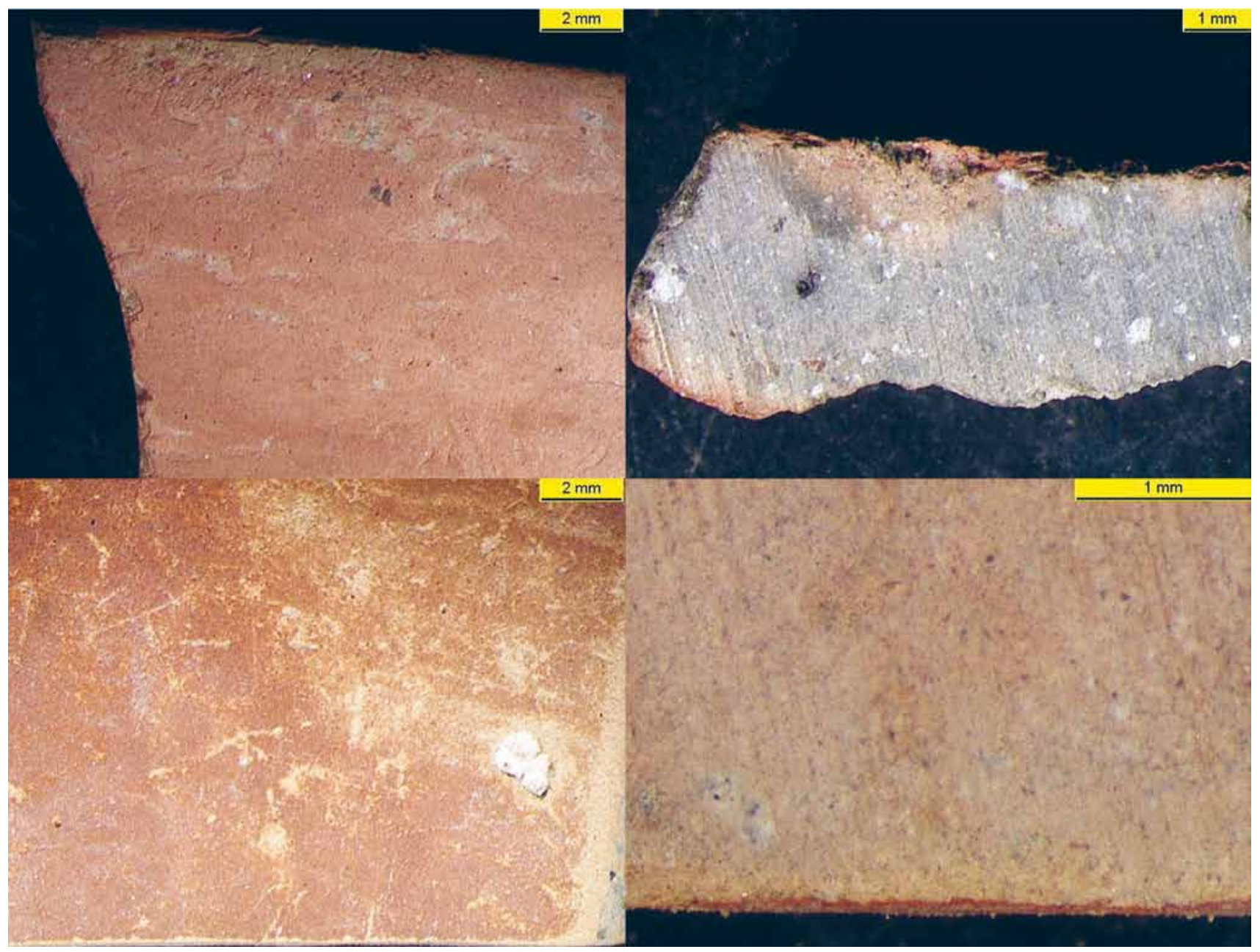

Abb. 8: Detail der Oberflächen außen (links) und im Querschnitt (rechts) der „unechten Terra Sigillata“ Keramik.

Fig. 8: Detail of the surface (left) and cross-section (right) of the "artificial Terra Sigillata"

als Malfixierungsmittel, als Alternativ zur Anwendung organischer und anorganischer Fixierungssubstanzen der Kaltbemalung, bewusst verwendet worden ist. Die Kaltbemalung ist für die Lengyelkeramik seit dem Anfang ihres Auftretens (um 4800 vor Chr.) und für ihre gesamte Dauer sehr kennzeichnend, während die Überzüge erst in einer eingehenden Phase der Produktion von Lengyelkeramik hergestellt worden sind. Nach den Keramikuntersuchungen von Michelstetten konnte festgestellt werden, dass das Auftreten der Herstellungstechnik der sogenannten „unechten Terra Sigillata“ Keramik zumindest ab 4600 vor Chr. nachgewiesen werden kann (CARNEIRo 2002: 37, 38, 123-130).

Interessant dabei ist, dass obwohl Überzüge wischfester sind und damit eine vorteilhafte Veränderung gegenüber der gebräuchlichen Maltechnologie der Lengyelkeramik darstellen, sie diese nicht ersetzt haben bzw. weitläufig viel weniger oft verwendet worden sind und nur auf die rote (möglicherweise z. Teil auch auf die schwarze) Farbe beschränkt sind. Die Kaltbemalung wurde unter Verwendung verschiedener Farben, neben der Erzeugung roter Überzüge, bis zur Endherstellung von Lengyelkeramik weiter angewendet. Darunter wurde die rote Farbe am häufigsten gebraucht. Für solchen Gebrauch konnten mehrere Argumente diskutiert werden. Als plausibler erscheint es mir, dass während die chemische Zusammensetzung von Hämatit (rote Farbe) erst bei $550^{\circ} \mathrm{C}$, ohne eine wesentliche Farbumwandlung zu er- leben, verändert wird, dagegen Jarosit bei $350-400^{\circ} \mathrm{C}$ und Gips bzw. Calciumssulfate bei $200^{\circ} \mathrm{C}$ schon veränderbar sind (Noll 1991: 187), wodurch die Haltbarkeit von Keramik mit weißen und gelben Überzügen und die gewünschte Färbung gefährdet würde. Andererseits, obwohl die Kaltbemalung weniger anhaltend als die mit Überzügen ist, stellt sie doch eine schnellere und einfacher herstellbare Technik dar, womit eine bunte, motivreiche Keramik erzeugt werden konnte, die den Zweck ihrer Hersteller/Benutzer erfüllt hat. Die Herstellung von hochglänzend polierter Keramik in den roten und schwarzen Farben könnte mit ihrem Gebrauch in bestimmten Situationen zusammenhängen. Dafür spricht auch im Allgemeinen die gute Ausführung ihrer Gefäße (meist aus weniger groben Herstellungsmaterialien, sorgfältig geformt und behandelt) und die Auswahl ihrer Formen, die zu einer betonten Profilierung neigen (Schüsseln mit ausladendem, gebogenem Oberteil; Töpfe und Flaschen aus weniger gewöhnlichen Formen).

\section{Literaturverzeichnis}

CARnEIro, Â. (2002): Studien zur Spätlengyelzeit am Beispiel von Keramik von Michelstetten (Niederösterreich). - Unpublizierte Dissertation der Universität Wien. - 560 S.; Wien.

Doneus, M. (2001): Die Keramik der mittelneolitischen Kreisgrabenanlage von Kamegg, Niederösterreich. Ein Beitrag zur Chronologie der Stufe MOG I der Lengyel-Kultur. - Mitteilungen der prähistorischen Kommission, 46: $146 \mathrm{~S}$. 
DRAžDÁK, K. (1973-74): Mineralogische Analyse des roten und gelben Farbstoffes der neolithischen Keramik (MBK) aus Těšetice-Kyjovice, Bez. Znaim. - Sborník Prací Filosofické Fakulty Brnenské Univerzity, E 18-19: 69-79.

GRILL, R. (1968): Erläuterungen zur geologischen Karte des nordöstlichen Weinviertels und zu Blatt Gänserndorf. - Geologische Bundesanstalt Wien - 155 S.; Wien (Verlag der Geologischen Bundesanstalt).

Hennicke, H. (1989): Rohstoffaufbereitung, Formgebung und Trocknen. In: SchneIdER, G.: Naturwissenschaftliche Kriterien und Verfahren zur Beschreibung von Keramik, - Acta Praehistorica et Archaeologica, 21: 15-18 S.(Anhang).

Kovarník, J. (1983-84): Zur Technologie der neolithischen Keramik. - Mitteilungen der österreichischen Arbeitsgemeinschaft für Ur- und Frühgeschichte, 33-34/1: 151-169 S.

Kovarník, J. (1989): Die Anwendung von mineralischen Farbstoffen im Neolithikum. - In: Rulf, J. (ed.): Bylany Seminar 1987. Collected papers: 149-160; Praha (Archeologický ústav).

Krenmayr, H.-G. \& Schnabel, W. (2002): Quartär - Ober - Pliozän (T1). -In: Schnabel, W. (ed.): Legende und Kürzerläuterung der Geologischen Karte von Niederösterreich 1:200.000, Geologische Bundesanstalt: 20-23 S.; Wien.
Neugebauer-Maresch, C. (1981): Archäologisches Fundmaterial aus den jungsteinzeitlichen Befestigungsanlagen Falkenstein-Schanzboden, NÖ. - Unpublizierte Dissertation der Universität Wien. - 255 S.; Wien.

Noll, W. (1991): Alte Keramiken und ihre Pigmente: Studien zu Material und Technologie. - 334 S.; Stuttgart (E. Schweizerbart'sche Verlagsbuchhandlung).

PALliardi, J. (1914): Die relative Chronologie der jüngeren Steinzeit in Mähren. - Wiener Prähistorischen Zeitschrift, 1: 1-24.

Roetzel, R. \& Schnabel, W. (2002): Molasse, Waschbergzone, Paläogen und Neogen auf der Böhmischen Masse, in W. Schnabel (red.), Legende und Kürzerläuterung der Geologischen Karte von Niederösterreich 1:200.000, Geologische Bundesanstalt, 23-30 S.; Wien.

Schnabel,W., Krenmayr, H.-G. \& Roetzel R. (2002): Geologie der Österreichischen Bundesländer, Niederösterreich. - Geologische Bundesanstalt, 2 Farbkarten und Erläuterungsbericht: 20-30.

Thenius, E. (1983): Niederösterreich im Wandel der Zeiten. Die Entwicklung der vorzeitlichen Tier- und Pflanzenwelt von Niederösterreich. - Katalog des Niederösterreichischen-Landesmuseums, NF 144: 156 S.

Wessely, G. (2006): Geologie der österreichischen Bundesländer, Niederösterreich. - Geologische Bundesanstalt, 416 S.; Wien.

Wosinsky, M. (1888): Das prähistorische Schanzwerk von Lengyel. Seine Erbauer und Bewohner. Heft 1: 221 S.; Budapest. 